\title{
Experiência de um hospital de referência com o retalho livre anterolateral da coxa na reconstrução de extremidades
}

Experience of a referral hospital with the free anterolateral thigh flap for extremity reconstruction

Experiencia de un hospital de referencia con el colgajo anterolateral libre del muslo en la reconstrucción de extremidades Nikolas Higa BENITES ${ }^{1}$

Guilherme Leipner MARGATHO²

Fernanda Ruiz de ANDRADE ${ }^{3}$

Luis Guilherme Rosifini Alves REZENDE ${ }^{4}$

Amanda Favaro CAGNOLATI ${ }^{4}$

Alex Eduardo Calderón IRUSTA 4

Nilton MAZZER ${ }^{5}$

${ }^{1}$ Ortopedista e Cirurgião de Mão, Microcirurgia e Membro Superior formado pelo Hospital das Clínicas da Faculdade de Medicina de Ribeirão Preto da Universidade de São Paulo (HC-FMRP-USP)14015-010 Ribeirão Preto-SP, Brasil

${ }^{2}$ Médico Residente em Ortopedia e Traumatologia do Hospital das Clínicas da Faculdade de Medicina de Ribeirão Preto da Universidade de São Paulo (HC-FMRP-USP) 14015-010 Ribeirão Preto-SP, Brasil

${ }^{3}$ Cirurgião de Mão do Hospital Estadual de Américo Brasiliense, parte do Complexo do Hospital das Clínicas da Faculdade de Medicina de Ribeirão Preto da Universidade de São Paulo (HC-FMRP-USP) 14015-010 Ribeirão Preto-SP, Brasil

${ }^{4}$ Médico Assistente da Divisão de Cirurgia da Mão, Microcirurgia e Membro Superior do Hospital das Clínicas da Faculdade de Medicina de Ribeirão Preto da Universidade de São Paulo (HC-FMRP-USP) 14015-010 Ribeirão Preto-SP, Brasil

${ }^{5}$ Professor Titular e chefe do Serviço de Cirurgia da Mão, Microcirurgia e Membro Superior do Hospital das Clínicas da Faculdade de Medicina de Ribeirão Preto da Universidade de São Paulo (HC-FMRP-USP) 14015-010 Ribeirão Preto-SP, Brasil

\section{Resumo}

Introdução: Com a evolução da microcirurgia ao longo dos anos o Retalho Anterolateral da Coxa vem se tornando uma das principais opções para reconstruções na cabeça, pescoço, tronco e extremidades devido sua versatilidade e confiabilidade. Objetivo: Descrever dados de um hospital terciário referência em trauma na reconstrução de extremidades com o Retalho Anterolateral da Coxa. Método: Este é um estudo retrospectivo de 18 retalhos Anterolateral da Coxa microcirúrgicos realizados entre Março de 2016 e Outubro de 2019 em pacientes de todas as idades, na reconstrução de membros, onde se observou dados referentes ao paciente: idade, sexo, membro acometido, tempo entre a lesão e a confecção do retalho; ao intraoperatório: anatomia dos vasos perfurantes, tempo cirúrgico total, vasos receptores utilizados; e informações do pós-operatório: número de cirurgias relacionadas ao retalho, necessidade de reabordagem e número de perdas. Foram excluídos pacientes que perderam seguimento ou que apresentaram dados do prontuário incompletos. Realizou-se estatística descritiva e cruzamento de algumas variáveis utilizando o teste t-Student. Resultados: Nas reconstruções houve predomínio de pacientes do sexo masculino (72\%), em idade produtiva, de etiologia traumática e nos membros inferiores. O tempo médio até a reconstrução foi de 21 dias e o tempo cirúrgico foi de 384 minutos. O paciente permaneceu, em média, 39 dias internado. Dos 18 retalhos, 3 evoluíram com necrose, 2 por trombose arterial e 1 por infecção. 6 retalhos necessitaram de reaborgadem de emergência, 3 por sangramento, 2 por congestão e 1 por infecção. Foram realizadas uma média de 3 cirurgias até a alta. Foram identificadas 15 perfurantes miocutâneas (83\%) e 3 septocutâneas (17\%). A análise do sucesso do retalho em relação ao tempo cirúrgico e dos dias até a cirurgia não mostrou significância estatística, assim como a necessidade de reabordagem em relação ao tempo cirúrgico. Conclusão: O retalho Anterolateral da Coxa mostrou-se confiável, além de apresentar diversas vantagens como: por ser retirado com uma grande ilha de pele, apresentar pedículo longo, vasos de bom calibre, não necessitar de mudança de decúbito e apresentar baixa morbidade da área doadora.

Descritores: Retalho Miocutâneo; Microcirurgia; Hospitais Especializados.

\section{Abstract}

Introduction: With the evolution of microsurgery over the years, the Anterolateral Thigh Flap has become one of the main option for reconstructions in the head, neck, trunk, and extremities due to its versatility and reliability. Objective: To describe data from a tertiary reference hospital in trauma in the reconstruction of extremities with the Anterolateral Thigh Flap. Method: This is a retrospective study of 18 microsurgical Anterolateral Thigh flaps performed between March 2016 and October 2019 in patients of all ages, in the reconstruction of limbs, where data related to the patient were observed: age, sex, affected limb, the time between the injury and the raising of the flap; intraoperative: anatomy of perforating vessels, total surgical time, recipient vessels used; and postoperative information: number of surgeries related to the flap, need for re-approach and number of losses. Patients who missed follow-up or who had incomplete medical record data were excluded. Descriptive statistics and cross-checking of some variables were performed using the t-Student test. Results: In reconstructions, male patients were predominant $(72 \%)$, of productive age, of traumatic etiology, and in the lower limbs. The average time to reconstruction was 21 days and the surgical time was 384 minutes. The patient remained in the hospital for an average of 39 days. Of the 18 flaps, 3 evolved with necrosis, 2 due to arterial thrombosis, and 1 due to infection. 6 flaps required emergency re-approach, 3 due to bleeding, 2 due to congestion, and 1 due to infection. An average of 3 surgeries was performed until hospital discharge. 15 myocutaneous perforators (83\%) and 3 septocutaneous perforators (17\%) were identified. The analysis of the success of the flap concerning the surgical time and the days until the surgery did not show statistical significance, either to re-approach concerning the surgical time. Conclusion: The Anterolateral Thigh flap proved to be reliable, in addition to presenting several advantages such as: being removed with a large island of skin, presenting a long pedicle, vessels of good caliber, not requiring a change of position and presenting low morbidity of the donor area.

Descriptors: Myocutaneous Flap; Microsurgery; Hospital, Special.

\section{Resumen}

Introducción: con la evolución de la microcirugía a lo largo de los años, el colgajo anterolateral del muslo se ha convertido en una de las principales opciones para reconstrucciones en la cabeza, el cuello, el tronco y las extremidades debido a su versatilidad y confiabilidad. Objetivo: describir los datos de un hospital de referencia terciario en trauma en la reconstrucción de extremidades con el colgajo anterolateral del muslo. Método: este es un estudio retrospectivo de 18 colgajos microquirúrgicos de muslo anterolateral realizados entre marzo de 2016 y octubre de 2019 en pacientes de todas las edades, en la reconstrucción de extremidades, donde se observaron datos relacionados con el paciente: edad, sexo, extremidad afectada, tiempo entre la lesión y la fabricación del colgajo; intraoperatorio: anatomía de los vasos perforantes, tiempo quirúrgico total, vasos receptores utilizados; e información postoperatoria: número de cirugías relacionadas con el colgajo, necesidad de reenfoque y número de pérdidas. Se excluyeron los pacientes que perdieron el seguimiento o que tenían datos de registros médicos incompletos. La estadística descriptiva y la verificación cruzada de algunas variables se realizaron mediante la prueba t-Student. Resultados: en las reconstrucciones, predominó el sexo masculino (72\%), la edad productiva, la etiología traumática y las extremidades inferiores. El tiempo promedio de reconstrucción fue de 21 días y el tiempo quirúrgico fue de 384 minutos. El paciente permaneció en el hospital durante un promedio de 39 días. De los 18 colgajos, 3 evolucionaron con necrosis, 2 debido a trombosis arterial y 1 debido a infección. 6 colgajos requirieron resurgimiento de emergencia, 3 debido a sangrado, 2 debido a congestión y 1 debido a infección. Se realizaron un promedio de 3 cirugías hasta el alta. Se identificaron 15 perforantes miocutáneos $(83 \%)$ y 3 perforadores septocutáneos $(17 \%)$. El análisis del éxito del colgajo en relación con el tiempo quirúrgico y los días hasta la cirugía no mostró significación estadística, así como la necesidad de volver a abordar en relación con el tiempo quirúrgico. Conclusión: el colgajo anterolateral del muslo demostró ser confiable, además de presentar varias ventajas como: ser extirpado con una gran isla de piel, presentar un pedículo largo, vasos de buen calibre, que no requieren un cambio de posición y una baja morbilidad del área donante. Descriptores: Microcirugia; Colgajo Miocutáneo; Hospitales Especializados

O tratamento de feridas complexas das extremidades constitui um grande desafio na rotina da cirurgia reconstrutiva. Desde a primeira descrição de um retalho "Groin flap" microcirúrgico por Daniel e Taylor em $1973^{1}$, a microcirurgia vem avançando e tornou-se prática comum em muitos serviços. Em 
termos práticos, essa evolução influenciou substancialmente na diminuição das amputações primárias de membros ${ }^{2}$.

Durante muitos anos, o retalho de Latíssimo do Dorso microcirúrgico foi a preferência para reconstrução de falhas de cobertura nas extremidades, principalmente no membro inferior, devido às suas vantagens: anatomia confiável, capacidade de cobertura de grandes defeitos, pedículo longo, mínima morbidade da área doadora e bom calibre dos $\operatorname{vasos}^{3,4}$.

A primeira descrição do retalho Anterolateral da Coxa foi feita em 1984 por Song e colaboradores e com o avanço da microcirurgia, esse retalho vêm ganhando espaço entre os microcirurgiões devido sua versatilidade, mesmo sendo tecnicamente mais desafiador ${ }^{5}$. Sua versatilidade se deve ao longo pedículo vascular, pela possibilidade de retirada de uma grande ilha de pele, possibilidade de levar um retalho com diferentes tipos de tecido associados e possuir uma baixa morbidade da área doadora ${ }^{6-8}$. Por ser obtido na maioria das vezes em decúbito dorsal facilita o trabalho em duas equipes, diminuindo o tempo cirúrgico, e também apresenta a vantagem de poder ser retirado fino, com a espessura de até 4 milímetros, o que não é possível com retalhos musculares, que são mais volumosos ${ }^{9,10}$.

Um dos principais fatores que o cirurgião considera na escolha de um retalho é a morbidade da área doadora e o retalho Anterolateral da Coxa gera um prejuízo funcional mínimo na coxa apresenta cicatriz em local menos visível que outros retalhos $^{11,12}$. Cicatrizes em locais muito visíveis, como o retalho Antebraquial, podem ser motivo de insatisfação do paciente ${ }^{13}$.

Dentre tantas opções de áreas doadoras de retalhos microcirúrgicos para o tratamento de falhas de cobertura, o retalho Anterolateral da Coxa vem se destacando com um dos principais recursos no arsenal do cirurgião reconstrutivo. $\mathrm{O}$ propósito desse trabalho foi descrever os resultados de um hospital terciário utilizando o retalho Anterolateral da Coxa no tratamento de pacientes que necessitam de cobertura microcirúrgica para preservação de um membro.

\section{MATERIAL E MÉTODO}

Trata-se de um estudo observacional retrospectivo de pacientes submetidos a reconstrução microcirúrgica do membro superior ou inferior com o retalho livre Anterolateral da Coxa no período de março de 2016 até outubro de 2019. O levantamento de dados foi feito através da revisão de prontuários de todos os pacientes submetidos a este procedimento no período especificado. Foram obtidos dados relativos ao paciente como: idade, sexo, membro acometido, tempo entre a lesão e a confecção do retalho; dados do intraoperatório: anatomia dos vasos perfurantes (septocutâneas ou miocutâneas), tempo cirúrgico total, vasos receptores utilizados; assim como informações do pós-operatório: número de cirurgias relacionadas ao retalho, necessidade de reabordagem e número de perdas. Como critérios de inclusão foram considerados os pacientes de todas as idades atendidos neste serviço que necessitaram do uso do retalho livre Anterolateral da Coxa para o tratamento de falha de cobertura nos membros inferiores ou superiores de qualquer etiologia. Foi considerado critério de exclusão os pacientes que perderam seguimento, bem como prontuários com dados incompletos.

Com os dados obtidos realizou-se estatística descritiva e também o cruzamento de algumas varáveis utilizando testes t-Student. Valores de $\mathrm{p}<0,05$ indicaram significância estatística. Todas as análises estatísticas foram realizadas com a utilização do software estatístico $\mathrm{SAS}^{14,15}$.

\section{RESULTADOS}

Foram realizados 18 retalhos em 17 pacientes (Tabela 1). Um paciente necessitou de dois retalhos para a cobertura da mesma lesão (casos $n^{\circ} 13$ e 14). Observou-se uma maior prevalência de indivíduos do sexo masculino (72\%) e da faixa etária de 21 a 40 anos (Figura 1).

Tabela 1. Lista dos retalhos em ordem cronológica de realização

\begin{tabular}{|c|c|c|c|c|c|c|c|c|c|c|c|}
\hline Retalho & Idade & Sexo & Defeito & IND & $\begin{array}{c}\text { TR } \\
\text { (Dias) }\end{array}$ & $\begin{array}{c}\text { TC } \\
\text { (Min) }\end{array}$ & RE & $\mathbf{s}$ & $\begin{array}{c}\text { PI } \\
\text { (Dias) }\end{array}$ & AA & PF \\
\hline 1 & 5 & $\mathrm{~F}$ & Dorso pé $\mathrm{E}$ & Trauma & 12 & 190 & $\mathrm{~N}$ & $\mathrm{~N}$ & 33 & $\mathrm{TA}$ & $\mathrm{MC}$ \\
\hline 2 & 55 & M & $\begin{array}{l}\text { Dorso mão } \\
\mathrm{E}\end{array}$ & Trauma & 15 & 535 & $\mathrm{~S}$ & $\mathrm{~N}$ & 85 & $\mathrm{RD}$ & $\mathrm{SC}$ \\
\hline 3 & 13 & M & $\begin{array}{l}1 / 3 \quad \text { distal } \\
\text { perna } D\end{array}$ & Trauma & 7 & 606 & $\mathrm{~N}$ & $\mathrm{~s}$ & 14 & $\mathrm{TP}$ & MC \\
\hline 4 & 23 & M & Calcâneo D & Trauma & 20 & 285 & $\mathrm{~N}$ & $\mathrm{~s}$ & 28 & TP & MC \\
\hline 5 & 19 & M & Maléolo LE & Trauma & 6 & 350 & $\mathrm{~S}$ & $\mathrm{~s}$ & 16 & TP & $\mathrm{SC}$ \\
\hline 6 & 25 & $\mathrm{~F}$ & $\begin{array}{l}\text { perna } E \\
\text { Lédio }\end{array}$ & Trauma & NA & 305 & $\mathrm{~N}$ & $\mathrm{~s}$ & 20 & TP & MC \\
\hline 7 & 30 & M & $\begin{array}{l}1 / 3 \text { distal } \\
\text { braço } \mathrm{E}\end{array}$ & Trauma & 17 & 440 & $\mathrm{~S}$ & $\mathrm{~s}$ & 45 & $\mathrm{BR}$ & MC \\
\hline 8 & 46 & M & Calcâneo E & Trauma & 17 & 480 & $\mathrm{~S}$ & $\mathrm{~N}$ & 37 & ТP & $\mathrm{MC}$ \\
\hline 9 & 32 & M & $\begin{array}{l}1 / 3 \text { distal } \\
\text { perna } \mathrm{E}\end{array}$ & Trauma & 5 & 369 & $\mathrm{~N}$ & $\mathrm{~S}$ & 13 & TP & MC \\
\hline 10 & 67 & M & $\begin{array}{l}1 / 3 \text { médio } \\
\text { perna } \mathrm{D}\end{array}$ & Trauma & 30 & 305 & $\mathrm{~N}$ & $\mathrm{~s}$ & 34 & ТР & MC \\
\hline 11 & 37 & M & $\begin{array}{l}1 / 3 \text { distal } \\
\text { perna } D\end{array}$ & Trauma & 27 & 500 & $\mathrm{~N}$ & $\mathrm{~S}$ & 32 & $\mathrm{TP}$ & MC \\
\hline 12 & 41 & M & $\begin{array}{l}1 / 3 \text { médio } \\
\text { perna } E\end{array}$ & Trauma & 12 & 350 & $\mathrm{~N}$ & $\mathrm{~S}$ & 24 & $\mathrm{TP}$ & MC \\
\hline 13 & 60 & $\mathrm{~F}$ & Dorso pé D & Trauma & 11 & 377 & $\mathrm{~S}$ & $\mathrm{~s}$ & 60 & TP & MC \\
\hline 14 & 60 & $\mathrm{~F}$ & Dorso pé D & Trauma & 24 & 359 & $\mathrm{~N}$ & $\mathrm{~s}$ & 60 & $\mathrm{TA}$ & MC \\
\hline 15 & 29 & M & $\begin{array}{l}1 / 3 \text { distal } \\
\text { antebraço } \\
\mathrm{D}\end{array}$ & Trauma & 8 & 455 & $\mathrm{~s}$ & $\mathrm{~S}$ & 35 & UL & MC \\
\hline 16 & 39 & M & $\begin{array}{l}\text { Dorso pé } \\
\text { direito }\end{array}$ & Trauma & 12 & 327 & $\mathrm{~N}$ & $\mathrm{~s}$ & 24 & $\mathrm{TP}$ & MC \\
\hline 17 & 40 & M & $\begin{array}{l}1 / 3 \text { médio } \\
\text { perna D }\end{array}$ & Trauma & 100 & 390 & $\mathrm{~N}$ & $\mathrm{~s}$ & 115 & TP & MC \\
\hline 18 & 65 & $\mathrm{~F}$ & Maléolo LD & Trauma & 36 & 297 & $\mathrm{~N}$ & $\mathrm{~s}$ & 37 & TP & $\mathrm{SC}$ \\
\hline
\end{tabular}

Fonte: Dados da pesquisa

$\mathrm{IND}=$ indicação; $\mathrm{TR}=$ tempo até retalho; $\mathrm{TC}=$ tempo cirúrgico; $\mathrm{RE}=$ reabordagem de emergência; $\mathrm{S}=$ sucesso; $\mathrm{PI}=$ período de internação; $\mathrm{AA}=$ anastomose arterial; $\mathrm{P}=$ perfurante; $\mathrm{RD}=$ radial; $\mathrm{BR}=$ braquial $\mathrm{UL}=$ ulnar; $\mathrm{TA}=$ tíbia anterior; $\mathrm{TP}=$ tíbia posterior; $\mathrm{MC}=$ miocutâneo; $\mathrm{SC}=$ septocutâneo; $\mathrm{E}=$ esquerdo $\mathrm{D}=$ direito; $\mathrm{LD}=$ lateral direito; $\mathrm{LE}=$ lateral esquerdo.

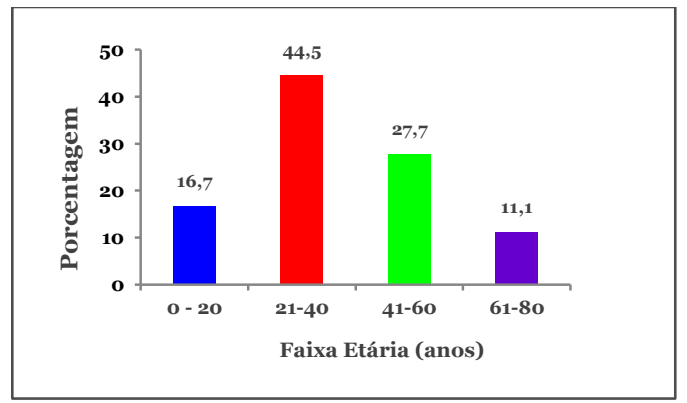

Figura 1: Gráfico de barras para a faixa etária de paciente com lesão traumática 
Para traçar o perfil epidemiológico de pacientes acometidos por trauma com importante lesão de partes moles excluiu-se o único caso de ressecção tumoral (caso ${ }^{\circ} 6$ ), mantendo uma maior prevalência de pacientes do sexo masculino e adultos jovens. Dentre as causas, 17 retalhos tiveram sua indicação devido a lesões traumáticas e apenas $1 \mathrm{em}$ ressecção tumoral. A maior parte foi utilizada para reconstrução de lesões nos membros inferiores (15 casos), sendo a perna o seguimento mais acometido.

Nas lesões traumáticas obtivemos uma média de 21 dias entre a data da lesão e a realização da cobertura e a média de tempo cirúrgico foi de 384 minutos, com uma variação de 190 a 606 minutos. Foram realizados em média 3 procedimentos cirúrgicos até a alta do paciente, englobando desde curativos até revisões de anastomose. Seis retalhos (33\%) necessitaram ser reabordados em caráter emergencial, 3 por sangramento (casos $n^{\circ} 5,7$ e 13), 2 por congestão (casos $n^{\circ} 2$ e 15$)$ e 1 por infecção aguda no pós-operatório (caso $\mathrm{n}^{\circ} 8$ ). Houve sucesso em $15(83 \%)$ dos retalhos realizados; entre as perdas, 2 foram por trombose arterial (caso $n^{\circ} 1$ e 2 ) e 1 por infecção. O tempo de internação médio foi de 39 dias, variando de 13 a 115 dias. Durante a dissecção do retalho, foram identificadas perfurantes miocutâneas em 15 casos (83\%) e septocutâneas em 3 casos (17\%).

Comparou-se a variável "sucesso do retalho" com o tempo cirúrgico e com o número de dias do trauma até a confecção do retalho, que não mostrou diferença estatística significativa. (Tabelas 2 e 3 ) Também não houve diferença estatística entre 0 tempo cirúrgico e a necessidade de reabordagem emergencial (Tabela 4).

\begin{tabular}{|c|c|c|c|c|c|c|}
\hline \multicolumn{7}{|c|}{ Tempo Cirúrgico (minutos) } \\
\hline SR & $\mathbf{n}$ & Média & Desvio-Padrão & Mínimo & Mediana & Máximo \\
\hline $\mathrm{N}$ & 3 & 401.67 & 185.36 & 190.00 & 480.00 & 535.00 \\
\hline $\mathrm{S}$ & 15 & 381.00 & 87.48 & 285.00 & 359.00 & 606.00 \\
\hline
\end{tabular}

Tabela 3. Relação entre o sucesso do retalho e o tempo até o retalho

\begin{tabular}{|c|c|c|c|c|c|c|}
\hline \multicolumn{7}{|c|}{ Tempo até o Retalho (dias) } \\
\hline SR & $\mathbf{n}$ & Média & Desvio-Padrão & Mínimo & Mediana & Máximo \\
\hline $\mathrm{N}$ & 3 & 14.67 & 2.52 & 12.00 & 15.00 & 17.00 \\
\hline S & 14 & 22.50 & 24.31 & 5.00 & 14.50 & 100.00 \\
\hline
\end{tabular}

Tabela 4. Relação entre tempo cirúrgico e reabordagem de emergência

\begin{tabular}{|l|l|l|l|l|l|l|}
\hline \multicolumn{7}{c}{ Tempo Cirúrgico (minutos) } \\
\hline RE & $\mathbf{n}$ & Média & Desvio-Padrão & Mínimo & Mediana & Máximo \\
\hline $\mathrm{N}$ & 12 & 356.92 & 107.33 & 190.00 & 338.50 & 606.00 \\
$\mathrm{~S}$ & 6 & 439.50 & 67.70 & 350.00 & 447.50 & 535.00 \\
\hline $\mathrm{RE}=$ & reabordagem de emergência & & & & \\
\hline
\end{tabular}

Teste t de Student: $\mathrm{p}$-valor = 0,0655 (t=-1.99; gl=14.8)

\section{DISCUSSÃO}

Os dados obtidos no presente estudo evidenciam que a maioria dos pacientes é do sexo masculino na proporção de 5,3 homens para cada mulher e em sua maioria, se encontravam em faixa etária economicamente ativa (dos 20 aos 40 anos), condizente com a literatura ${ }^{16,17}$.Também se observou uma predominância de reconstruções nos membros inferiores $(83 \%)$, principalmente para cobertura da tíbia que é o osso com maior incidência de fraturas expostas $^{18}$.

As perfurantes cutâneas do retalho surgem do ramo descendente da artéria circunflexa femoral lateral, entre o espaço intermuscular do reto femoral e do vasto lateral. Dos 18 retalhos realizados, 3 vasos eram septocutâneos (17\%) e 15 miocutâneo (83\%) proporção semelhante a observada por Kuo et al. ${ }^{8} \mathrm{e}$ Kimata et al. ${ }^{12}$. Não houve casos em que o vaso perfurante estava ausente, mesmo havendo uma pequena porcentagem de pacientes descrita na literatura, que não apresentam a perfurante cutânea. Este fato é um dos principais motivos para que este retalho seja temido por alguns cirurgiões, apesar de todas suas outras vantagens 8 .

Em relação ao tempo cirúrgico, nenhum paciente necessitou de mudança de decúbito e todos os casos foram operados com 2 equipes, estando sempre presente ao menos 1 cirurgião Sênior. Atribuiu-se a grande variação no tempo cirúrgico pelo fato de os casos terem sido operados em um hospital-escola, onde as mudanças cíclicas da equipe somadas ao preceito do ensino podem ser importantes influenciadores desses dados ${ }^{19}$. Observou-se um tempo cirúrgico médio menor nos casos em que houve sucesso do retalho e nos casos que não necessitaram de reabordagem de emergência, porém, eles não apresentaram diferença estatística significativa, com $\mathrm{p}=0,866$ e $\mathrm{p}=0,065$, respectivamente.

Em 1986 Godina $^{20}$ realizou um importante estudo que evidenciou uma alta taxa de sucesso do retalho naqueles pacientes submetidos a reconstrução precoce até 72 horas do trauma $(99,25 \%)$, com a piora desse índice nas reconstruções "atrasadas" (entre 72 horas e 3 meses) e "tardias" (após 3 meses) ${ }^{20}$. Nesta série de casos viu-se que, das lesões traumáticas, apenas um paciente encontra-se no grupo das reconstruções "tardias" enquanto os demais estão no grupo das reconstruções "atrasadas", alcançando uma taxa de sucesso de $82 \%$, próxima a taxa de $88 \%$ encontrada por Godina ${ }^{20}$ em suas reconstruções "atrasadas". Ao usar o teste t de Student para relacionar o tempo até o retalho com o sucesso da microcirurgia não obteve-se resultado confiável visto que a amostragem está concentrada apenas no grupo das reconstruções "atrasadas".

Sabe-se que a maior parte das complicações, principalmente trombose venosa e/ou arterial, ocorrem nas primeiras 24 horas de pós-operatório e podem ser revertidas em grande parte dos $\operatorname{casos}^{21}$. Nesta série de casos, 6 retalhos tiveram que ser revisados em caráter de emergência com uma taxa de 
sucesso das revisões de $66,5 \%$.

O tempo médio de internação dos pacientes foi de 39 dias, sendo que o maior deles foi de 115 dias devido à perda de um retalho Latíssimo do Dorso (caso $\mathrm{n}^{\circ} 17$ ) feito previamente. O tempo de internação prolongado foi condizente com os achados de Godina ${ }^{20}$ que também relacionou o tempo até a realização da cirurgia reconstrutiva com o aumento dos dias de internação.

\section{CONCLUSÃO}

Demonstrou-se que esta amostra é predominantemente de pacientes do sexo masculino, jovens e com falha de cobertura no membro inferior. Obteve-se sucesso em $82 \%$ dos casos. O tempo médio entre o trauma e a cirurgia de cobertura foi de 21 dias, considerado atrasado, o que demonstra as dificuldades encontradas em um hospital público do interior do estado. O Retalho Anterolateral da Coxa mostrou ser extremamente confiável nas mãos de um cirurgião experiente por poder ser retirada uma grande ilha de pele, com ou sem músculo, apresentar pedículo longo, vasos de bom calibre, não necessitar de mudança de decúbito e apresentar baixa morbidade da área doadora.

\section{REFERÊNCIAS}

1. Daniel RK, Taylor GI. Distant transfer of an island flap by microvascular anastomoses. A clinical technique. Plast Reconstr Surg. 1973;52(2):111-17.

2. Ninkovic M, Voigt S, Dornseifer U, Lorenz S, Ninkovic M. Microsurgical advances in extremity salvage. Clin Plast Surg. 2012;39(4):491-505.

3. Tamimy MS, Rashid M, Ehtesham-ul-Haq, Aman S, Aslam A, Ahmed RS. Has the anterolateral thigh flap replaced the latissimus dorsi flap as the workhorse for lower limb reconstructions? J Pak Med Assoc. 2010; 60(2):76-81.

4. Spyropoulou A, Jeng SF. Microsurgical coverage reconstruction in upper and lower extremities. Semin Plast Surg. 2010;24(1):34-42.

5. Xiong L, Gazyakan E, Kremer T, Hernekamp FJ, Harhaus L, Saint-Cyr M et al. Free flaps for reconstruction of soft tissue defects in lower extremity: a meta-analysis on microsurgical outcome and safety. Microsurgery. 2016; 36(6):511-24.

6. Song YG, Chen GZ, Song YL. The free thigh flap: a new free flap concepted based on the septocutaneous artery. Br J Plast Surg. 1984; 37(2):149-59.

7. Wei FC, Jain V, Celik N, Chen HC, Chuang DC, Lin $\mathrm{CH}$. Have we found an ideal soft-tissue flap? An experience with 672 anterolateral thigh flaps. Plast Reconstr Surg. 2002;109(7):2219-26
8. Kimata Y, Uchiyama K, Ebihara S, Nakatsuka T, Harii K. Anatomic variation and technical problems of the anterolateral thigh flap: A report of 74 cases. Plast Reconstr Surg. 1998; 102(5):1517-23

9. Spindler N, Al-Benna S, Ring A, Homann H, Steinsträsser L, Steinau HU et al. Free anterolateral thigh flaps for upper extremity soft tissue reconstruction. GMS Interdiscip Plast Reconstr Surg DGPW. 2015;4:Doc05.

10. Kimura N, Satoh K, Hasumi T, Ostuka T. Clinical application of the free thin anterolateral thigh flap in 31 consecutive patients. Plast Reconstr Surg. 2001;108(5):1197-210.

11. Collins J, Ayeni O, Thoma A. A systematic review of anterolateral thigh flap donor site morbidity. Can J Plast Surg. 2012;20(1):17-23.

12. Kuo YR, Jeng SF, Kuo MH, Huang MN, Liu YT, Chiang YC et al. Free anterolateral thigh flap for extremity reconstruction: Clinical experience and functional assessment of donor site. Plast Reconstr Surg. 2001;107(7):1766-71

13. Novak CB, Lipa JE, Noria S, Allison K, Neligan PC, Gilbert RW. Comparison of anterolateral thigh and radial forearm free flap donor site morbidity. Microsurgery. 2007;27(8):651-54.

14. Pagano M, Gauvreau K. Princípios de Bioestatística. São Paulo: Pioneira Thomson Learning; 2004.

15. SAS Institute Inc., SAS/STAT® User's Guide. Version 9.4. Cary, NC: SAS Institute Inc.

16. Arruda LRP, Silva MAC, Malerba FG, Turíbio FM, Fernandes MC, Matsumoto MH. Fraturas expostas: estudo epidemiológico e prospectivo. Acta ortop bras. 2009;17(6):326-30.

17. Cunha FM, Braga GF, Drumond Jr SN, Figueiredo CTO. Epidemiologia de 1.212 fraturas expostas. Rev Bras Ortop. 1998;33(6):451-56.

18. Court-Brown CM, Rimmer S, Prakash U, McQueen MM. The epidemiology of open long bone fractures. Injury. 1998;29(7):529-34.

19. Shabtai M, Rosin D, Zmora O, Munz Y, Scarlat A, Shabtai EL et al. The impact of a resident's seniority on operative time and length of hospital stay for laparoscopic appendectomy: outcomes used to measure the resident's laparoscopic skills. Surg Endosc. 2004;18(9):1328-30.

20. Godina M. Early microsurgical reconstruction of complex trauma of the extremities. Plast Reconstr Surg.1986;78:285-292

21. Smit JM, Acosta R, Zeebregts CJ, Liss AG, Anniko M, Hartman EH. Early reintervention of compromised free flaps improves success rate. Microsurgery. 2007;27(7):612-16. 


\section{CONFLITO DE INTERESSES}

Os autores declaram não haver conflitos de interesse.

\section{AUTOR PARA CORRESPONDÊNCIA}

Fernanda Ruiz de Andrade

fernandaruiz90@hotmail.com

Submetido em 31/12/2019

Aceito em 11/01/2020 